\title{
VARIETIES WITH COFINAL SETS: EXAMPLES AND AMALGAMATION
}

\author{
PETER BRUYNS AND HENRY ROSE
}

(Communicated by Andreas R. Blass)

\begin{abstract}
A variety $\mathscr{V}$ has a cofinal set $S \subset \mathscr{V}$ if any $A \in \mathscr{V}$ is embeddable in a reduced product of members of $S$. Amalgamation in and examples of such varieties are considered. Among other results, the following are proved: (i) every lattice is embeddable in an ultraproduct of finite partition lattices; (ii) if $\mathscr{V}$ is a residually small, congruence distributive variety whose members all have one-element subalgebras, then the amalgamation class of $\mathscr{V}$ is closed under finite products.
\end{abstract}

\section{INTRODUCTION}

A set $S$ of members of a variety $\mathscr{V}$ is said to be cofinal in $\mathscr{V}$ if every $A \in \mathscr{V}$ is embeddable in a reduced product of members of $S$. Given a cofinal set $S \subset \mathscr{V}$ we let $E_{S}(\mathscr{V})$, or simply $E(\mathscr{V})$, be the class of all reduced products of members of $S$ modulo some (usually specified) type of filter. We shall refer to $E(\mathscr{V})$ as the envelope of $\mathscr{V}$ determined by $S$ and the type of filter involved. In this paper we only deal with cases where the filter involved is either trivial (that is $E(\mathscr{V})=P(S)$ is just the class of all products of members of $S$ ) or an ultrafilter. A variety $\mathscr{V}$ is said to be ultra-universal if there is $U \in \mathscr{V}$ such that every $A \in \mathscr{V}$ is embeddable in an ultrapower of $U$. In this case we refer to $U$ as an ultra-universal member of $\mathscr{V}$. Clearly $U$ is ultra-universal if and only if the following holds: for any universal sentence $\phi, U \models \phi$ implies $A \models \phi$ for any $A \in \mathscr{V}$. Among the most obvious examples of ultra-universal varieties are group and lattice varieties or, more generally, varieties of algebras whose members have one-element subalgebras.

If $\mathscr{V}$ is a residually small variety, the collection of all (maximal) subdirectly irreducible members of $\mathscr{V}$ forms a cofinal set for $\mathscr{V}$ since every member of $\mathscr{V}$ is embeddable into a product of members of this cofinal set. Obviously not every such $\mathscr{V}$ is ultra-universal.

Received by the editors December 14, 1988 and, in revised form, March 1, 1990.

1980 Mathematics Subject Classification (1985 Revision). Primary 03C05, 03C20, 06B20, 08B10, 08B25.

The work of both authors was supported by grants from the University of Cape Town Research Committee. That of the second author was also supported by a grant from the South African Council for Scientific and Industrial Research. 
The central result of this paper is Theorem 1.2 which tells us that if $\mathscr{V}$ is a residually small, congruence distributive variety and every member of $\mathscr{V}$ has a one-element subalgebra then the amalgamation class of $\mathscr{V}$ is closed under finite products.

Examples of ultra-universal algebras can be found in the last section. In particular in this section it is shown that every lattice is embeddable in an ultraproduct of finite partition lattices.

\section{Amalgamation in Residually SMall, CONGRUENCE-DISTRIBUTIVE VARIETIES WHOSE MEMBERS ALL HAVE ONE-ELEMENT SUBALGEBRAS}

The amalgamation class of variety was introduced in [6] and studied in [11]. The residually small varieties were characterized in [10]. We assume throughout that $\mathscr{V}$ is a variety as in the title of this section. We use the following notation (see $[2,8]$ for more details):

$\operatorname{Amal}(\mathscr{V})$ - the amalgamation class of $\mathscr{V}$.

$\mathscr{V}_{S I}\left(\mathscr{V}_{M I}\right)$-the set of (maximal) subdirectly irreducible members of $\mathscr{V}$.

We have the following:

Theorem 1.1 (Jipsen and Rose [7]). (i) For $A \in \mathscr{V}$ the following are equivalent.

(a) $A \in \operatorname{Amal}(\mathscr{V})$.

(b) For any embedding $f: A \rightarrow B \in \mathscr{V}$ and any homomorphism $g: A \rightarrow$ $M \in \mathscr{V}_{M I}$ there is a homomorphism $h: B \rightarrow M$ such that $g=h f$.

(ii) Any product of members of $\mathscr{V}_{M I}$ is an absolute retract in $\mathscr{V}$ and therefore belongs to $\operatorname{Amal}(\mathscr{V})$.

Using this result we are going to prove the following:

Theorem 1.2. $\operatorname{Amal}(\mathscr{V})$ is closed under finite products.

The proof of the following result can be found in [8].

Lemma 1.3. If $A=A_{\beta} \times A_{\gamma}$ is a product of members of a congruence distributive variety and $s$ is a homomorphism defined on $A$ then there are homomorphisms $s_{\beta}$ and $s_{\gamma}$ on $A_{\beta}$ and $A_{\gamma}$ such that $s=\left\langle s_{\beta}, s_{\gamma}\right\rangle$.

For our next lemma consider a product $A=A_{\beta} \times A_{\gamma}$ where $A_{\beta}, A_{\gamma} \in$ $\operatorname{Amal}(\mathscr{V})$ and $\mathscr{V}$ is a variety as in the title of this section. Let $B=\prod M_{i}$ be a product of members of $\mathscr{V}_{M I}$ and consider an embedding $f: A \rightarrow B$. The one-element subalgebra embeddings $\bar{\beta}: A_{\beta} \rightarrow A$ and $\bar{\gamma}: A_{\gamma} \rightarrow A$ induce embeddings $f_{\beta}=f \bar{\beta}: A_{\beta} \rightarrow B$ and $f_{\gamma}=f \bar{\gamma}: A_{\gamma} \rightarrow B$. Consider an embedding $g=\left\langle f_{\beta}, f_{y}\right\rangle: A \rightarrow B^{2}$.

Lemma 1.4. The diagram $\left(A, f, B, g, B^{2}\right)$ can be amalgamated in $\mathscr{V}$.

Proof. Let $p: B \rightarrow B^{2}$ be an embedding given by $p(b)=\langle b, b\rangle$ for all $b \in B$ and put $k=p f: A \rightarrow B^{2}$. To prove the lemma it is sufficient to show that the 
diagram $\left(A, g, B^{2}, k, B^{2}\right)$ can be amalgamated in $\mathscr{V}$. We shall use Lemma 2.4; that is, we are going to show that for $u \neq v \in B^{2}$, there are homomorphisms $\sigma$ and $\tau$ on $B^{2}$ such that $\sigma(u) \neq \sigma(v)$ and $\tau g=\sigma k$. So let $u \neq v \in B^{2}$ with $u=\left\langle b_{1}, b_{2}\right\rangle$ and $v=\left\langle c_{1}, c_{2}\right\rangle$ where $b_{1}, b_{2}, c_{1}, c_{2} \in B$. Since $u \neq v$ we may assume $b_{1} \neq c_{1}$. Then $\left(b_{1}\right)_{i} \neq\left(c_{1}\right)_{i}$ for some $i$. Let $\pi: B \rightarrow M=M_{i}$ be the $i$ th projection and consider an epimorphism $\sigma=\langle\pi, \pi\rangle: B^{2} \rightarrow M^{2}$. Then $\sigma(u) \neq \sigma(v)$. Let $s=\sigma k: A \rightarrow M^{2}$. By Lemma 1.3 there are homomorphisms $s_{\beta}$ and $s_{\gamma}$ on $A_{\beta}$ and $A_{\gamma}$ such that $s=\left\langle s_{\beta}, s_{\gamma}\right\rangle$, that is for $a \in A$ with $a=$ $\left\langle a_{\beta}, a_{\gamma}\right\rangle$ we have $s(a)=\left\langle s_{\beta}\left(a_{\beta}\right), s_{\gamma}\left(a_{\gamma}\right)\right\rangle$. Since $s(A) \leq \sigma p(B) \cong M$, we may assume that $s_{\beta}: A_{\beta} \rightarrow M$ and $s_{\gamma}: A_{\gamma} \rightarrow M$. Now $f_{\beta}: A_{\beta} \rightarrow B$ and $f_{\gamma}: A_{\gamma} \rightarrow B$ are embeddings and so by Theorem 3.1 (i) there are homomorphisms $t_{\beta}: B \rightarrow M$ and $t_{\gamma}: B \rightarrow M$ such that $t_{\gamma} f_{\beta}=s_{\beta}$ and $t_{\gamma} f_{\gamma}=s_{\gamma}$. Let $\tau=\left\langle t_{\beta}, t_{\gamma}\right\rangle: B^{2} \rightarrow M^{2}$. Then for $a \in A$ with $a=\left\langle a_{\beta}, a_{\gamma}\right\rangle$ we have $\tau g(a)=\tau\left[\left\langle f_{\beta}\left(a_{\beta}\right), f_{\gamma}\left(a_{\gamma}\right)\right\rangle\right]=$ $\left\langle t_{\beta} f_{\beta}\left(a_{\beta}\right), t_{\gamma} f_{\gamma}\left(a_{\gamma}\right)\right\rangle=\left\langle s_{\beta}\left(a_{\beta}\right), s_{\gamma}\left(a_{\gamma}\right)\right\rangle=s(a)=\sigma k(a)$.

Proof of Theorem 1.2. Let $A$ be a product of finitely many members of $\operatorname{Amal}(\mathscr{V})$. Without loss of generality we may assume that $A$ is a product of two factors. It follows that $P\left(\mathscr{V}_{M I}\right)$ forms an envelope of $\mathscr{V}$ which is determined by the set $\mathscr{V}_{M I}$. The theorem follows then from Theorem 1.1 (ii), Theorem 2.8, and Lemma 1.4.

Let $A=\prod_{\gamma \in \alpha} A_{\gamma}$ be a product of members $\operatorname{Amal}(\mathscr{V})$ where $\mathscr{V}$ is a variety as in Theorem 1.2. For $\gamma \in \alpha$ let $e_{\gamma}$ be an arbitrary but fixed element of $A_{\gamma}$ such that $\left\{e_{\gamma}\right\}$ is a one-element subalgebra of $A_{\gamma}$. Let $\bar{A}$ be a subalgebra of $A$ described as follows:

For $a \in A$ with $a=\left\langle a_{\gamma}\right\rangle_{\gamma \in \alpha}$, the element $a \in \bar{A}$ iff $\left\{a_{\gamma}: a_{\gamma} \neq e_{\gamma}\right\}$ is finite.

Corollary 1.4. $\bar{A} \in \operatorname{Amal}(\mathscr{V})$.

Proof. Let $X$ be the set of all finite subsets of $\alpha$ and, for $x \in X$ with $x=$ $\left\{\gamma_{1}, \ldots, \gamma_{n}\right\}$, let $A_{x}=A_{\gamma_{1}} \times \cdots \times A_{\gamma_{n}}$. Then $\bar{A}$ is a union of an updirected family $\left\{A_{x}: x \in X\right\}$. By Theorem $1.2, A_{x} \in \operatorname{Amal}(\mathscr{V})$ for each $x \in X$. Since every updirected union of members of $\operatorname{Amal}(\mathscr{V})$ belongs to $\operatorname{Amal}(\mathscr{V})$ (see [11]), we have that $\bar{A} \in \operatorname{Amal}(\mathscr{V})$.

\section{AMALgamation IN SET COFINAL VARIETIES}

Lemma 2.1. Let $E(\mathscr{V})$ be an envelope determined by some cofinal set of a variety $\mathscr{V}$. Then for $A \in \mathscr{V}$ the following are equivalent.

(1) $A \in \operatorname{Amal}(\mathscr{V})$.

(2) Any two embeddings of $A$ into $B, C \in E(\mathscr{V})$ can be amalgamated in $\mathscr{V}$.

Proof. Clearly (1) implies (2). Suppose (2) holds and let $\left(A, f, B^{\prime}, g, C^{\prime}\right)$ be a diagram in $\mathscr{V}$. Then, since for some $B, C \in E(\mathscr{V})$ we have $B^{\prime} \leq B$ and 
$C^{\prime} \leq C$, it follows from (2) that the above diagram can be amalgamated in $\mathscr{V}$.

Call an envelope $E(\mathscr{V})$ updirected if, for any $B, C \in E(\mathscr{V})$, there is $D \in$ $E(\mathscr{V})$ such that $B \leq D$ and $C \leq D$.

Example 2.2. If $U$ is an ultra-universal member of a variety $\mathscr{V}$ then an envelope $E(\mathscr{V})$ which consists of all ultrapowers of $U$ is updirected. In order to show this we need some model-theoretic results which can be found in [4].

Consider a pair of ultrafilters $G$ and $F$ over sets $I$ and $J$ respectively. Define $G \times F$ to be the set of all $Y \subset I \times J$ such that

$$
\{j \in J:\{i \in I:\langle i, j\rangle \in Y\} \in G\} \in F .
$$

Then $G \times F$ is an ultrafilter over $I \times J$. Further, if $\left\{A_{i j}:\langle i, j\rangle \in I \times J\right\}$ is a set of models, then the ultraproduct $\prod_{G \times F} A_{i j}$ and the ultraproduct of ultraproducts $\prod_{F}\left(\prod_{G} A_{i j}\right)$ are isomorphic. Now the statement about updirectedness of $E(\mathscr{V})$ will follow immediately since both ultrapowers $\prod_{G} U$ and $\prod_{F} U$ are embeddable in $\prod_{F}\left(\prod_{G} U\right)$.

Corollary 2.3. Let $E(\mathscr{V})$ be an updirected envelope of a set cofinal variety $\mathscr{V}$. Then for $A \in \mathscr{V}$ the following are equivalent.

(i) $A \in \operatorname{Amal}(\mathscr{V})$.

(ii) Any two embeddings of $A$ into $B \in \mathscr{V}$ can be amalgamated in $\mathscr{V}$.

(iii) Any two embeddings of $A$ into $C \in E(\mathscr{V})$ can be amalgamated in $\mathscr{V}$.

Proof. Clearly (i) implies (ii) and (ii) implies (iii). It follows from Lemma 2.1 and the updirectedness of $E(\mathscr{V})$ that (iii) implies (i).

For the next result we need the following.

Lemma 2.4 (Grätzer and Lakser [6]). A diagram $(A, f, B, g, C)$ in a variety $\mathscr{V}$ can be amalgamated if and only if for all $u \neq v \in B$ there exists $D \in \mathscr{V}$ and homomorphisms $f^{\prime}: B \rightarrow D, g^{\prime}: C \rightarrow D$ such that $f^{\prime} f=g^{\prime} g$ and $f^{\prime}(u) \neq$ $f^{\prime}(v)$, and the same holds for $C$.

Let $E(\mathscr{V})$ be an updirected envelope of a set cofinal variety $\mathscr{V}$. For $B \in$ $E(\mathscr{V})$ let $B_{S I}$ be the set of all subdirectly irreducible images of $B$.

Corollary 2.5 (cf. [2, Lemma 3.8]). Let $A \in \mathscr{V}$ and suppose that for any embedding $f: A \rightarrow B \in E(\mathscr{V})$ and any homomorphism $k: A \rightarrow K \in B_{S I}$ there is a homomorphism $f^{\prime}: B \rightarrow K$ such that $k=f^{\prime} f$. Then $A \in \operatorname{Amal}(\mathscr{V})$.

Proof. By Corollary 2.3 we have to show that any two embeddings $f: A \rightarrow B$ and $g: A \rightarrow B$ with $B \in E(\mathscr{V})$ can be amalgamated in $\mathscr{V}$. We shall use Lemma 2.4: let $u \neq v \in B$. Then there is an epimorphism $g^{\prime}: B \rightarrow K \in B_{S I}$ such that $g^{\prime}(u) \neq g^{\prime}(v)$.We have $g^{\prime} g: A \rightarrow K$. By assumption there is a homomorphism $f^{\prime}: B \rightarrow K$ with $g^{\prime} g=f^{\prime} f$. This completes the proof.

Call a variety $\mathscr{V}$ factor-embeddable if it satisfies the following property. If $A=\prod_{\gamma \in \alpha} A_{\gamma}$ is a product of members of $\mathscr{V}$, then for any $\gamma \in \alpha$ there is an 
embedding $\bar{\gamma}: A_{\gamma} \rightarrow A$ such that for $a_{\gamma} \in A_{\gamma}$ the $\gamma$ th coordinate of $\bar{\gamma}\left(a_{\gamma}\right)$ is $a_{\gamma}$.

Remark 2.6. If $\mathscr{V}$ is a variety whose members all have one-element subalgebras then $\mathscr{V}$ is factor-embeddable. In fact every factor-embeddable variety is ultra-universal.

For our next lemma we let $A=\prod_{\gamma \in \alpha} A_{\gamma}$ be a product of members of the amalgamation class of a factor-embeddable variety $\mathscr{V}$.

Given embeddings $f: A \rightarrow B$ and $g: A \rightarrow C$ with $B, C \in \mathscr{V}$, consider embeddings $h: A \rightarrow B^{\alpha}$ and $k: A \rightarrow C^{\alpha}$ such that $h=\left\langle f_{\alpha}\right\rangle_{\gamma \in \alpha}$ and $k=\left\langle g_{\alpha}\right\rangle_{\gamma \in \alpha}$ where, for $\gamma \in \alpha, f_{\gamma}=f \bar{\gamma}: A_{\gamma} \rightarrow B$ and $g_{\gamma}=g \bar{\gamma}: A_{\gamma} \rightarrow B$.

Lemma 2.7. The diagram $\left(A, h, B^{\alpha}, k, C^{\alpha}\right)$ can be amalgamated in $\mathscr{V}$.

Proof. Since for $\gamma \in \alpha$, we have $A_{\gamma} \in \operatorname{Amal}(\mathscr{V})$, each diagram $\left(A_{\gamma}, f_{\gamma}, B, g_{\gamma}\right.$,

$C$ ) has an amalgam $\left(D_{\gamma}, s_{\gamma}, t_{\gamma}\right)$. Put $D=\prod_{\gamma \in \alpha} D_{\gamma}$ and consider embeddings:

$$
\begin{aligned}
& \left\langle s_{\gamma}\right\rangle_{\gamma \in \alpha}=s: B^{\alpha} \rightarrow D, \\
& \left\langle t_{\gamma}\right\rangle_{\gamma \in \alpha}=t: B^{\alpha} \rightarrow D .
\end{aligned}
$$

Then for $a \in A$ with $a=\left\langle a_{\gamma}\right\rangle_{\gamma \in \alpha}$ we have $\operatorname{sh}(a)=s\left[\left\langle f_{\gamma}\left(a_{\gamma}\right)\right\rangle_{\gamma \in \alpha}\right]=\left\langle s_{\gamma} f_{\gamma}\left(a_{\gamma}\right)\right\rangle_{\gamma \in \alpha}$ $=\left\langle t_{\gamma} g_{\gamma}\left(a_{\gamma}\right)\right\rangle_{\gamma \in \alpha}=s\left[\left\langle g_{\gamma}\left(a_{\gamma}\right)\right\rangle_{\gamma \in \alpha}\right]=s k(a)$. Thus $(D, s, t)$ is an amalgam of $\left(A, h, B^{\alpha}, g, C^{\alpha}\right)$.

For our next result we let $E(\mathscr{V})$ be an envelope of a factor-embeddable variety $\mathscr{V}$ and assume that $P(E(\mathscr{V})) \subset \operatorname{Amal}(\mathscr{V})$, that is every product of members of $E(\mathscr{V})$ belongs to the amalgamation class of $\mathscr{V}$. An example of such $\mathscr{V}$ can be found in the next section.

Theorem 2.8. Let $A=\prod_{\gamma \in \alpha} A_{\gamma}$ be a product of members of $\operatorname{Amal}(\mathscr{V})$. Then $A \in \operatorname{Amal}(\mathscr{V})$ if and only if for any two embeddings $f: A \rightarrow B \in E(\mathscr{V})$ and $g: A \rightarrow C \in E(\mathscr{V})$ and induced embeddings $h: A \rightarrow B^{\alpha}$ and $k: A \rightarrow C^{\alpha}$, as in the preceding lemma, the diagrams $\left(A, f, B, h, B^{\alpha}\right)$ and $\left(A, g, C, k, C^{\alpha}\right)$ can be amalgamated in $\mathscr{V}$.

Proof. It follows from Lemma 2.1 that to prove the nontrivial implication we have to show that the diagram $(A, f, B, g, C)$ can be amalgamated in $\mathscr{V}$. By Lemma 2.5 the diagram $\left(A, h, B^{\alpha}, k, C^{\alpha}\right)$ has an amalgam $(F, d, e)$ (see Figure 1). Without loss of generality we may assume that $F \in E(\mathscr{V})$ so that $F \in \operatorname{Amal}(\mathscr{V})$. By assumption the diagrams $\left(A, f, B, h, B^{\alpha}\right)$ and $\left(A, g, C, k, C^{\alpha}\right)$ have amalgams $\left(X, f^{\prime}, h^{\prime}\right)$ and $\left(Y, g^{\prime}, k^{\prime}\right)$ and, since $P(E(\mathscr{V})) \subset \operatorname{Amal}(\mathscr{V})$, the diagrams $\left(B^{\alpha}, e, F, h^{\prime}, X\right)$ and $\left(C^{\alpha}, d, F, k^{\prime}, Y\right)$ have amalgams $(G, s, t)$ and $(H, u, v)$. Let $(K, p, q)$ be an amalgam of $(F, u, H, s, G)$. Then $\left(K, q t f^{\prime}, p v g^{\prime}\right)$ is an amalgam of $(A, f, B, g, C)$.

\section{ULTRA-UNIVERSAL VARIETIES}

For our next two examples we need a classical result concerning the embedding of an algebra into an ultraproduct of its finitely generated subalgebras. We 


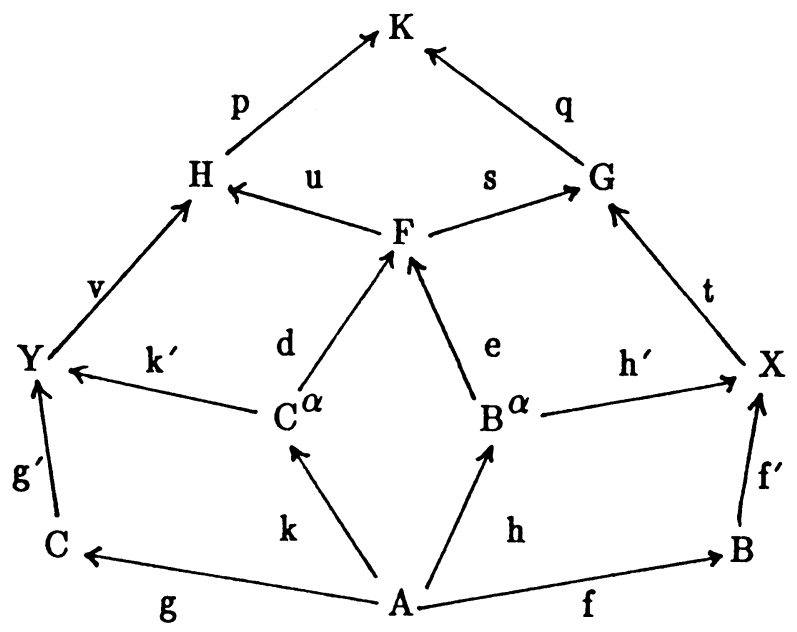

FIGURE 1

first introduce the concept of a finite subset filter.

Let $X$ be the family of all finite subsets of a set $Y$. For each $x \in X$ set $J_{x}=\{z \in X: z \supset x\}$. Then the family

$$
\left\{J_{x}: x \in X\right\}
$$

has the finite intersection property. We shall refer to any filter of $Y$ which contains the family $(*)$ as a finite subset filter in $Y$.

The proof of the following result can be found in [3, p. 213].

Lemma 3.1. Every algebra $A$ is embeddable in an ultraproduct of its finitely generated subalgebras modulo a finite subset filter of $A$.

With the help of this lemma we may show that certain algebras are ultrauniversal.

Example 3.2. Every infinite permutation group is ultra-universal. Indeed, let $G$ be an infinite permutation group. Then every finitely generated group is embeddable in $G$. Thus by Lemma 3.1, every group is embeddable in an ultrapower of $G$.

Remark 3.3. By Theorem 3.5 below every infinite partition lattice is ultrauniversal. This together with Example 3.2 implies that if $\varphi$ is a universal or existential sentence then $\varphi$ holds in one infinite permutation group (partition lattice) iff $\varphi$ holds in any infinite permutation group (partition lattice).

Example 3.4. Let $\mathscr{V}$ be a variety which satisfies the following property.

If $A=\prod_{i \in I} A_{i}$ is a product of members of $\mathscr{V}$ then $A_{i}$ is embeddable in $A$ for each $i \in I$. 
Then $\mathscr{V}$ is ultra-universal. Indeed, since the collection of all nonisomorphic, finitely generated members of a variety forms a set, we let $A$ be a product of members of this set. It follows from Lemma 3.1 that every member of $\mathscr{V}$ is embeddable in an ultrapower of $A$.

The following result provides examples of ultra-universal lattices.

Theorem 3.5. Let $\mathscr{V}$ be the variety of all lattices. Then the following lattices are ultra-universal in $\mathscr{V}$.

(i) Every infinite partition lattice.

(ii) A sublattice of compact elements of an infinite partition lattice.

(iii) A reduced product of finite partition lattices modulo a finite subset filter of an infinite set.

(iv) A nontrivial lattice $A$ with the following property: there is an infinite partition lattice $L$ such that every positive sentence holding in $L$ holds in $A$.

The finite subset filter of a set was discussed in the beginning of this section and, for basic properties of partition lattices (= lattices of equivalence relations), the reader is referred to [5].

Let $L$ be a partition lattice over an infinite set $Y$, and let $I$ be a sublattice of compact elements of $L$. Denote by $X$ the set of all finite subsets of $Y$. For $x \in X$ let $\rho_{x} \in I$ be a partition given as follows: the only nontrivial block of $\rho_{x}$ is $x$. Then the principal ideal $\left(\rho_{x}\right]$ in $I$ (and in $\left.L\right)$ is isomorphic to a finite partition lattice over $x$. Consider the direct product

$$
A=\prod_{x \in X}\left(\rho_{x}\right]
$$

Let $G$ be a finite subset filter of $Y$ and denote by $A / G$ the reduced product of $\left\{\left(\rho_{x}\right]: x \in X\right\}$ modulo $G$.

Lemma 3.6. $I$ is embeddable in $A / G$.

Proof. For $a \in I$ choose $\rho_{x}$ such that $a \in\left(\rho_{x}\right]$ and consider $\bar{a} \in A$ such that $\bar{a}(z)=a$ for all $z \in J_{x}$. Then the mapping $a \rightarrow \bar{a} / G$ (where $\bar{a} / G$ is a congruence class modulo $G$ ) is an embedding of $I$ into $A / G$.

To prove Theorem 3.5 we need the following observation due to $\mathrm{K}$. Baker (see [5, p. 223]).

$(* *) \quad$ If a universal sentence holds for all finite lattices then it holds
for all lattices.

Proof of Theorem 3.5. Let $I$ be the sublattice of the compact elements of an infinite partition lattice $L$ and let $K$ be any lattice. Suppose that $\varphi$ is a universal sentence which holds in $I$. Since every finite partition lattice is embeddable in $I$ and every finite lattice is embeddable in a finite partition lattice (see [9]), it follows from (**) that $\varphi$ holds in $K$. Thus $K$ is embeddable in an ultrapower of $I$ (see [1, p. 187, Lemma 3.8]), and so (ii) holds. Further, 
(iii) holds by Lemma 3.6 and (i) holds since $I$ is a sublattice of $L$. Proof of (iv): Let $A$ and $L$ be as in (iv). Then there are elementary extensions $A^{\prime}$ and $L^{\prime}$ such that $A^{\prime}$ is an image of $L^{\prime}$ (see [4, p. 236, Proposition 5.5.12]). Since $A$ and hence $A^{\prime}$ are nontrivial, this homomorphism embeds $L$ into $A^{\prime}$. This is because the top and bottom elements of $L^{\prime}$ are the top and bottom elements of its elementary submodel $L$. Thus every universal sentence which holds in $A$ must hold in $L$ and therefore $L$ is embeddable in an ultrapower of $A$. Since any lattice $K$ is embeddable in an ultrapower of $L$ we have that $K$ is embeddable in an ultrapower of an ultrapower of $A$. Now (iv) follows since this "double ultrapower" is isomorphic to an ultrapower of $A$ (see Example 2.2).

As a corollary to Theorem 3.5 we have

Theorem 3.7. Every lattice is embeddable in an ultraproduct of finite partition lattices.

Proof. Let $K$ be a lattice and $I$ be a sublattice of compact elements of an infinite partition lattice. We may assume that a filter $G$ from Lemma 3.6 is an ultrafilter so that $I$ is embeddable in an ultraproduct $\prod_{G}\left(\rho_{x}\right]$ of finite partition lattices. By Theorem 3.5 (ii) there is an ultrafilter $F$ such that $K$ is embeddable in the ultrapower $\prod_{F} I$, whence $K$ is embeddable in $\prod_{F}\left(\prod_{G}\left(\rho_{x}\right]\right)$. The theorem follows since the lattice $\prod_{F}\left(\prod_{G}\left(\rho_{x}\right]\right)$ is isomorphic to the ultraproduct $\prod_{G \times F}\left(\rho_{x}\right]$ (see Example 2.2).

\section{ACKNOWLEDGMENTS}

The second author wishes to thank his students C. Naturman, P. Ouwehand and A. Salamon for their lively discussions concerning this paper.

\section{REFERENCES}

1. J. L. Bell and A. B. Slomson, Models and ultraproducts, North-Holland, 1969.

2. C. Bergman, Amalgamation classes of some distributive varieties, Algebra Universalis 20 (1985), 143-166.

3. S. Burris and H. P. Sankapanavar, A course in universal algebra, Springer-Verlag, 1981.

4. C. Chang and H. J. Keisler, Model theory, North-Holland, 1973.

5. G. Grätzer, General lattice theory, Birkhäuser Verlag, Basel and Stuttgart, 1978.

6. G. Grätzer and H. Lakser, The structure of pseudocomplemented, distributive lattices II: Congruence extensions and amalgamations, Trans. Amer. Math. Soc. 156 (1971), 343-358.

7. $\mathrm{P}$. Jipsen and $\mathrm{H}$. Rose, Absolute retracts and amalgamation in certain congruence-distributive varieties, Canad. Math. Bull. 32 (1989), 309-313.

8. B. Jónsson, Amalgamation in small varieties of lattices, preprint, 1986.

9. P. Pudlak and J. Tuma, Every finite lattice can be embedded in the lattice of all equivalences over a finite set, Algebra Universalis 10 (1980), 74-95.

10. W. Taylor, Residually small varieties, Algebra Universalis 2 (1972), 33-53.

11. M. Yasuhara, The amalgamation property, the universal-homogeneous models and the generic models, Math. Scand. 34 (1974), 5-36. 